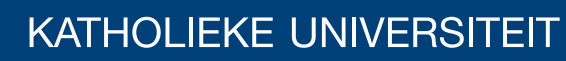 \\ LEUVEN
}

\section{Faculty of Business and Economics}

\$IDWDUHHG IKHXUUMF IRUMFKHGXOQJIP RGXOUSLRVAFW

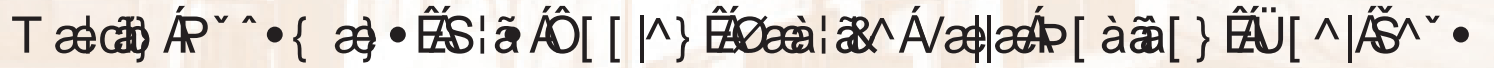

DEPARTMENT OF DECISION SCIENCES AND INFORMATION MANAGEMENT (KBI) 


\title{
A fast greedy heuristic for scheduling modular projects
}

\author{
Martijn Huysmans*, Kris Coolen*, Fabrice Talla Nobibon*‡, Roel Leus*§
}

\begin{abstract}
This article describes a heuristic for scheduling so-called 'modular' projects. Exact solutions to this NP-hard problem can be obtained with existing branch-and-bound and dynamic-programming algorithms, but only for small to medium-size instances. The proposed heuristic, by contrast, can be used even for large instances, or when instances are particularly difficult because of their characteristics, such as a low network density. The proposed heuristic draws from existing results in the literature on sequential testing, which will be briefly reviewed. The performance of the heuristic is assessed over a dataset of 360 instances.
\end{abstract}

Keywords: scheduling; greedy heuristics; modular projects.

\section{Introduction}

During Research and Development (R\&D) projects, activities can fail to meet their expected outcomes. An activity's failure can result in the termination of the entire project, for instance when a proposed new drug fails a toxicity test. In many other cases, however, the failure of one activity is not fatal to the project, because alternative activities are available that pursue the same target. When designing a new car, for example, several slightly different designs may be 'in the running'. If the preferred one of these designs fails the wind-tunnel test, the project could simply move on to the next one in line. Activities that have the same target are said to constitute a module. When one of the activities in a module is successful, the module is completed and the project can continue with the next module. In order to successfully complete the project, all the modules need to achieve success. Only when all of the scheduled activities in a module fail, does the project fail.

In our problem statement, we will allow for precedence constraints to be specified both within and between modules. Precedence constraints between modules can be regulatory or technical in nature. Regulatory constraints often occur to protect testers or consumers; when testing a new drug, for instance, the toxicity has to be tested (e.g., via animal testing) before clinical tests on humans are allowed [7]. An example of a technical constraint is the impossibility of evaluating a new car design in the wind tunnel before a prototype is manufactured for this test. Precedence constraints within modules are used to model situations where one activity cannot be tried before the other. In pharmaceuticals, for example, when a module aims to prove the effectiveness of a drug, a first job may be measuring the effects after one week. If no desired effects have been found after one week, one could either decide to give up the project, or to go on to an alternative activity, for instance waiting for the results to show up after two weeks. Alternatively, trials may be repeated in different doses and with different test subjects, etc.

Projects such as those described above will be referred to as modular projects. Exact scheduling algorithms for this type of projects have recently been developed by Coolen et al. [5]. When only few precedence constraints are imposed, however, these algorithms either run out of memory or become very slow when the number of activities increases. The goal of this paper is to develop a heuristic that produces 'good' schedules for such projects, i.e., schedules with a high expected profit, while requiring only very limited CPU time and computer memory. The results will be compared with the optimal solutions obtained by the algorithms described in [5].

The remainder of this text is organized as follows: in Section 2, the modular project scheduling problem on one machine is defined. In the development of a fast heuristic for this problem, we will draw from the literature on sequential testing; we therefore include a discussion of the similarities

\footnotetext{
${ }^{*}$ ORSTAT, Faculty of Business and Economics, KU Leuven, Leuven, Belgium.

${ }^{\ddagger}$ Postdoctoral Fellow of the Research Foundation - Flanders (FWO).

${ }^{\S}$ Corresponding author. E-mail: Roel.Leus@kuleuven.be. Postal address: ORSTAT, Faculty of Business and Economics, KU Leuven, Naamsestraat 69, B-3000 Leuven, Belgium. Tel. +32163269 67. Fax +32 16326624 .
} 
with this branch of literature and of its most relevant references in Section 3. The main contribution of this text is the greedy algorithm presented in Section 4, and we substantiate its computational performance by means of a series of experiments that are summarized in Section 5 . We conclude in Section 6.

\section{Problem description}

Most of the notation and definitions in this section are in line with [5].

\subsection{Definitions}

A project consists of a set of jobs $N=\{1, \ldots, n\}$ to be scheduled (the terms 'job' and 'activity' are used interchangeably), and we additionally define jobs 0 and $n+1$ as a dummy start and end job. The set of all jobs, including dummy jobs, is denoted by $\bar{N}=N \cup\{0, n+1\}$. Each job belongs to a unique module $i \in \bar{M}=\{0,1, \ldots, m+1\}$, with $\bar{M}$ the set of modules. The symbol $N_{i}$ denotes the set of jobs that belong to module $i$. The artificial start and end jobs 0 and $n+1$ are the sole jobs of modules 0 and $m+1$, respectively: $N_{0}=\{0\}$ and $N_{m+1}=\{n+1\}$. The set of non-dummy modules is denoted by $M=\{1, \ldots, m\}$. Since each job belongs to exactly one module, the set of all $N_{i}$ constitutes a partition of $\bar{N}$.

Every job $k \in N$ has a probability of technical success $p_{k}$. The probability of failure will be denoted by $q_{k}=1-p_{k}$. The probabilities $p_{k}$ are gathered in vector $\mathbf{p}$ (with $p_{k}$ as its $k$-th component). Each job $k \in N$ also has an associated cost $c_{k}$, which is the $k$-th component of vector c. The dummy end job $n+1$ has a positive payoff $V$ instead of a cost; this payoff is only collected when the overall project succeeds, i.e., when for each module at least one job was successful.

The precedence constraints between the modules are defined by a partial order $\bar{A}$ over $\bar{M}$. This partial order relation is a collection of predecessor-successor pairs $(i, j)$ : module $j$ has as a prerequisite the successful execution of module $i$ if and only if $(i, j) \in \bar{A}$. Relation $\bar{A}$ induces the relation $A$ on $M$. The precedence constraints between jobs belonging to the same module $i$ are described by the partial order $B_{i}$ : job $l$ in module $i$ has as a precondition that job $k$ in module $i$ must be attempted first if and only if $(k, l) \in B_{i}$. Finally, for practical reasons, all precedence-related activity pairs are collected in one 'induced' order relation $B^{*} \subset N \times N$ :

$$
(k, l) \in B^{*} \quad \Leftrightarrow \quad\left(\left(\exists i \in M:(k, l) \in B_{i}\right) \quad \vee \quad\left(\exists(i, j) \in A:\left(k \in N_{i}\right) \wedge\left(l \in N_{j}\right)\right)\right) .
$$

With these definitions, $\left(N, B^{*}\right)$ is a partially ordered set or poset, and the same holds for $(M, A)$ and $\left(N_{i}, B_{i}\right)$ for all $i \in M$. An illustration of the precedence constraints that can apply to a scheduling instance is provided in Figure 1; we graphically represent these constraints by means of a two-layered network, where modules are rounded boxes and activities are round nodes. For this instance, $n=3, m=2, A=\emptyset, B_{1}=\{(1,2)\}$ and $B_{2}=\emptyset$. Including modules 0 and $m+1$ in the analysis, we have $\bar{A}=\{(0,1),(0,2),(0,3),(1,3),(2,3)\}$. Note that transitive arcs between modules, such as $(0,3)$, are omitted from the figure.

Essential to the problem description is the uncertainty in the activity outcomes. A realization or state vector $\mathbf{x}=\left(x_{1}, \ldots, x_{n}\right) \in\{0,1\}^{n} \equiv \mathbb{B}^{n}$ is a vector indicating for each job $k$ whether it succeeds $\left(x_{k}=1\right)$ or fails $\left(x_{k}=0\right)$. Each component $x_{k}$ is the outcome of a Bernoulli trial $X_{k}$ with probability of success $p_{k}$. The random variables $X_{1}, \ldots, X_{n}$ are assumed to be mutually independent. The actual realization $x_{k}$ of each $X_{k}$ is known only after job $k$ is executed. The symbol $\mathbf{X}$ represents the vector with components $X_{k}$. 


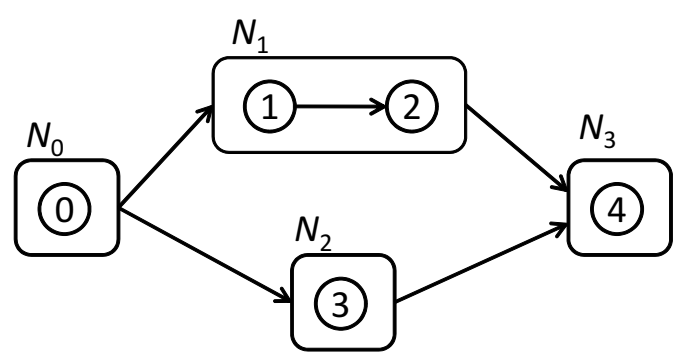

Figure 1: Graphical representation of a modular project with five jobs and four modules.

For scheduling, the only relevant characteristics of the jobs are the following: their probability of success, their cost, and their predecessors. In this paper, we only consider 'sequential' schedules, in which jobs are processed one-at-a-time, and we therefore do not explicitly consider job durations. As pointed out in $[6,7]$, when cash flows (costs and payoff) are not discounted, or more generally when the cash flows are time-independent, then it is a dominant decision to not schedule jobs in parallel. An alternative reason for restricting the analysis to sequential schedules only is the possible use of a scarce (bottleneck) resource that can perform only one job at a time, e.g., expensive equipment or highly-skilled labor. Therefore, a schedule $\mathbf{s}$ is an ordered subset of the activities of a scheduling instance. By $s_{t}$ we denote the job in position $t$. A job can only be scheduled when all the preceding modules have been successfully completed and all preceding jobs in the same module have been attempted. Schedules that respect these precedence constraints are called feasible; the set of schedules that is feasible under scenario $\mathbf{x}$ is denoted by $\Sigma_{\mathbf{x}}$. The set of all feasible schedules is given by $\Sigma=\bigcup_{\mathbf{x} \in \mathbb{B}^{n}} \Sigma_{\mathbf{x}}$.

\subsection{Problem statement}

The problem under study is to maximize the expected profit of a modular project by deciding which jobs to schedule and in which order, taking into account the precedence constraints. The payoff is only obtained when the project is completed successfully. The project success function for our setting is defined as:

$$
\sigma(\mathbf{x}, \mathbf{s})= \begin{cases}1 & \text { if } \mathbf{s} \text { is successful for } \mathbf{x} \\ 0 & \text { otherwise }\end{cases}
$$

where $\mathbf{s}$ is a schedule that is feasible for $\mathbf{x}$. We define $\mathbf{s}$ to be successful for scenario $\mathbf{x}$ if for every module there is at least one job scheduled that turns out to be successful, i.e.,

$$
\forall i \in M: \exists k=s_{t} \in N_{i} \text { with } x_{k}=1 .
$$

The project's profit is given by

$$
F(\mathbf{x}, \mathbf{s})=\sigma(\mathbf{x}, \mathbf{s}) V-\sum_{i=1}^{|s|} c_{s_{i}} .
$$

Due to the inherent uncertainty, a solution to this scheduling problem is not a single schedule but rather a scheduling policy, which is a set of rules that dynamically decide which scheduling decisions to make (which new jobs to start) based on the observed history of the project. A policy $\Pi$ can be seen as a function $\Pi: \mathbb{B}^{n} \rightarrow \Sigma$, mapping scenarios $\mathbf{x}$ to schedules $\mathbf{s}=\Pi(\mathbf{x})$ that are feasible with respect to $\mathbf{x}$. Alternatively, a policy can be represented by a binary decision tree, where each node represents an activity and each pair of branches emanating from a node represents failure (left branch) or success (right branch) of the corresponding activity; the root node of the tree decides on 


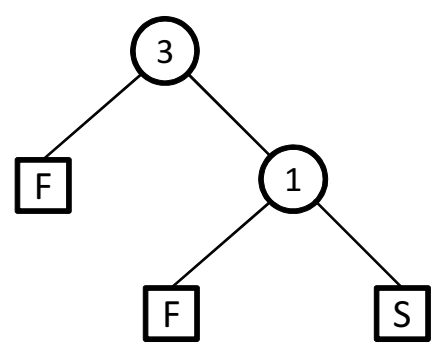

Figure 2: A scheduling policy for the project in Figure 1.

the first job to schedule. In this way, the schedule $\mathbf{s}=\Pi(\mathbf{x})$ for an arbitrary scenario $\mathbf{x}$ corresponds to a unique path in the binary decision tree from the root node down to a leaf node. An example of a scheduling policy for the project with precedence network as in Figure 1 is given in Figure 2. The leaf nodes are marked ' $\mathrm{F}$ ' for failure or ' $\mathrm{S}$ ' for success. This policy starts with job 3 of module 2 . If job 3 fails, module 2 fails and the project fails as well. If it succeeds, job 1 is scheduled. If this job succeeds, module 1 is completed and the project is finished successfully. This policy will never schedule job 2, so if job 1 fails, module 1 fails and the entire project is abandoned (which means no more activities are scheduled, and the payoff is not obtained). In other words, the issue of job selection is inherent in the problem statement.

Problem MP1 ('Modular Project scheduling on One Machine') can now be formally stated as follows: from a given class of policies $\mathcal{C}$, select a policy $\Pi^{*}$ that maximizes the expected profit of the project:

$$
\Pi^{*}=\underset{\Pi \in \mathcal{C}}{\arg \max } \mathbb{E}[F(\mathbf{X}, \Pi(\mathbf{X}))],
$$

with $\mathbb{E}[\cdot]$ the expectation operator. An instance of MP1 can by described by its parameters $\left(M, A,\left(N_{i}, B_{i}\right)_{i \in M}, \mathbf{p}, \mathbf{c}, V\right)$. This problem has been proved to be NP-hard, even for certain restrictive special cases [5]. This means that, unless $\mathrm{P}=\mathrm{NP}$, no exact polynomial-time algorithm exists, which motivates our search for an efficient heuristic that provides good solutions to MP1 instances in polynomial time.

\subsection{Classes of scheduling policies}

Various classes of scheduling policies are presented in [5]; the definitions below mainly draw from that source. We will restrict our solution space to only one class, namely the 'elementary modulesequence policies'.

We first describe the class $\mathcal{C}_{E}$ of elementary policies. An elementary policy is characterized by a compatible ordering $L$ of a subset of $N$. A compatible ordering is an ordering that respects all precedence constraints, which means that $L$ should be a linear extension of the subgraph of $\left(N, B^{*}\right)$ induced by $L$. From this ordering $L$ and for every scenario, a schedule is generated as follows: start with the first job in $L$, if it fails and there are no more jobs of its module left in $L$, abandon the project. If the job succeeds, this means the module is completed and all other jobs from this module can be deleted from $L$. Iterating through $L$ in this way, the schedule $\mathbf{s}$ is then simply given by the jobs that where considered.

Another class of interest to us is that of the module-wise policies $\mathcal{C}_{M}$. A module-wise policy is a policy that only produces schedules in which all jobs belonging to the same module are executed consecutively, or informally: there is no 'jumping' between modules. A module-sequence policy then, is a module-wise policy that adheres to the same linear extension of the module order $A$ for each possible realization. Finally, policies that are both elementary and module-sequence are called 
elementary module-sequence policies or EMS. The class of EMS policies is denoted by $\mathcal{C}_{E M S}$. The policy depicted in Figure 2, for example, is an EMS policy, characterized by the ordering $L=(3,1)$.

In this paper, we will restrict our search to EMS policies because of several reasons. First, they have a compact representation - their ordering $L$ - that is easy to interpret and work with. Second, their expected profit can be evaluated in linear time. Third, there always exists an EMS policy that is optimal in the larger class $\mathcal{C}_{E} \cup \mathcal{C}_{M}$, even though EMS policies are less in number. There is, however, an important caveat: EMS policies are not globally optimal. This means that there exist MP1 instances for which no elementary policy is optimal. EMS policies can even turn out to be arbitrarily bad vis-à-vis the global optimum; more precisely, there are instances for which an optimal EMS policy is defined by the empty list (with zero profit) whereas binary decision trees defining non-elementary policies with strictly positive expected profit exist. Even though the worst-case performance of an optimal EMS policy may be arbitrarily bad, the average performance over a large dataset of 'typical' instances turns out to be quite good: in [5], the average difference between globally optimal policies and optimal elementary policies was below $0.01 \%$ (less than one hundredth of a percent). Unfortunately, finding an optimal elementary policy has turned out to be quite time-consuming, which is why in this text we focus on finding good heuristic EMS policies.

\section{Sequential testing problems}

In this section, we review a number of concepts and results from the sequential testing literature that can be transposed to the MP1 setting. The heuristic for MP1, to be described in Section 4, is based on an optimal procedure for testing so-called 'series-parallel' systems.

\subsection{Link between testing and scheduling}

A review of the literature on sequential testing can be found in Ünlüyurt [11]. In testing problems, one is concerned with the system function $f$ rather than with the Boolean project success function as used in MP1, for a system consisting of a number of components. The system function $f$ indicates the state of the system for every possible combination of states of the underlying components. The state of the components is given by a vector $\mathbf{x}$, with $x_{i}=1$ if component $i$ is working, and $x_{i}=0$ if it is failing - we deliberately re-define some symbols, to underline their equivalence in the two settings (scheduling and testing); obviously, we equate activities in the scheduling problem with components in the testing problem. When the system is working, $f(\mathbf{x})=1$. When it is failing, $f(\mathbf{x})=0$. Each of the state variables $x_{i}$ is the outcome of a Bernoulli variable $X_{i}$ with probability of success $p_{i}$. The probability that the component fails is given by $q_{i}=1-p_{i}$.

The Boolean system function that has the closest ties to our MP1 scheduling problem is given by:

$$
f(\mathbf{x})=\bigwedge_{i \in M}\left(\bigvee_{k \in N_{i}} x_{k}\right) .
$$

This system function corresponds to a series-parallel system of depth 2. A series-parallel system (SPS) can be defined recursively as a system that consists of a serial or parallel connection of subsystems that are themselves SPS [11]. The depth of an SPS is defined as $1+\max$ \{depth of proper subsystem\}. For example, (simple) serial and parallel systems can be thought of as SPSs of depth 1 , with system function $f(\mathbf{x})=\wedge_{i} x_{i}$, respectively $f(\mathbf{x})=\vee_{i} x_{i}$. There are two types of SPSs of depth 2, which are named according to their global structure. A two-level SPS that is a serial connection (respectively parallel arrangement) of parallel (respectively serial) subsystems, is also simply called a series-parallel (respectively parallel-series) system [2]. 
A solution to a testing instance is also a policy $\Pi$, which maps each state vector $\mathbf{x} \in \mathbb{B}^{n}$ to a schedule s. A major difference between testing and scheduling, however, is the following: when testing, one will continue exploring new components until the actual state of the system is known with certainty. This means that, regardless of the order in which components are tested, the final output will always be the actual state of the system. In MP1, on the other hand, one can abandon the project prematurely - the result being that the project fails, even though it may have been possible to complete it successfully had more activities been undertaken, but the remaining activities may simply be too costly compared to the expected payoff. As a consequence, a scheduling policy $\Pi$ may fail to identify $f(\mathbf{x})$ for certain scenarios $\mathbf{x} \in \mathbb{B}^{n}$, the result being that $\sigma(\mathbf{x}, \Pi(\mathbf{x}))=0$ even though $f(\mathbf{x})$ may have been equal to 1 .

The second difference between testing and scheduling is the objective function. When testing, the goal is to minimize the expected cost of discovering the state of the system. The cost function $G(\mathbf{s})$ for schedule $\mathbf{s}$ is given by $\sum_{i \in \mathbf{s}} c_{i}$. Depending on the context, this can represent a financial cost or the time needed to test the components, or another measure of testing effort. A generic statement of the sequential testing problem, in line with our problem statement in Section 2.2, is then:

$$
\Pi^{*}=\underset{\Pi \in \mathcal{T}}{\arg \min } \mathbb{E}[G(\Pi(\mathbf{X}))]
$$

where $\mathcal{T}$ is a given class of testing policies. When no precedence constraints apply to the sequencing of the component tests and with a system function of the form (2), we have the unconstrained seriesparallel sequential testing problem, denoted USPST for short.

The concept of an elementary policy can also be defined for testing problems. In light of the impossibility of early abandonment (discussed supra), however, the compatible ordering $L$ defining an elementary policy now needs to be an ordering of the entire component set $N$. One minor modification can be made, namely that $L$ needs to contain only the non-redundant components of the instance, where a component $k$ is redundant if it has no influence on the system, that is $\forall \mathbf{x} \in \mathbb{B}^{n}$ with $x_{k}=0$ we have $f(\mathbf{x})=f\left(\mathbf{x} \vee e_{k}\right)$, with $e_{k}$ the $k$-th unit vector of $\mathbb{R}^{n}$. Without loss of generality, we will simply work with an ordering of $N$ below. We thus conclude that the class $\mathcal{C}_{E}$ of elementary scheduling policies contains the class $\mathcal{T}_{E}$ of elementary testing policies, for the same parameters.

\subsection{Optimality results for specific sequential testing problems}

In this section, we discuss a number of sequential testing problems without precedence constraints for which elementary policies are globally optimal. For a simple series system, the order $(1,2, \ldots, n)$ is optimal if and only if $\frac{c_{1}}{q_{1}} \leq \frac{c_{2}}{q_{2}} \leq \ldots \leq \frac{c_{n}}{q_{n}}$. The proof relies on a straightforward interchange argument, and has been provided by Mitten in 1960 [10] and by Butterworth in 1972 [4]; for discussions of this result, see for example $[2,11]$. This result can be transferred to MP1: an elementary policy defined by the same ordering is optimal for an MP1 instance with each $\left|N_{i}\right|=1$ and no precedence constraints (at least if $V$ is large enough, otherwise the empty list is optimal).

Similarly, for a simple parallel system the order $(1,2, \ldots, n)$ is optimal if and only if $\frac{c_{1}}{p_{1}} \leq \frac{c_{2}}{p_{2}} \leq$ $\ldots \leq \frac{c_{n}}{p_{n}}$. This is also an ordering defining an optimal elementary policy for MP1 instances with $|M|=1$ and $B_{1}=\emptyset$, but the jobs $k$ (at the end of the list) that satisfy the following test should be removed (see [5] for a discussion):

$$
c_{k}>p_{k} V
$$

The optimal testing procedure for a series-parallel system can be derived using a two-stage approach based on the results above, following Ben-Dov [2]. First, order the components within each parallel subsystem $i$ (the testing equivalent of a module) according to the optimal testing 
procedure for parallel systems; denote the obtained order by $L_{i}$. Subsystem $i$ as a whole then, has an associated probability of failure $\theta_{i}=\prod_{k \in N_{i}} q_{k}$ and an associated expected cost

$$
\kappa_{i}\left(L_{i}\right)=\sum_{k=1}^{\left|L_{i}\right|}\left(\prod_{l=1}^{k-1} q_{[l]_{L_{i}}}\right) c_{[k]_{L_{i}}}
$$

where empty products are taken to be 1 . The symbol $[k]_{\alpha}$ denotes the object in the $k$-th position according to permutation $\alpha$. When it is clear from the context, the permutation to be used - in this case $L_{i}$ - will be omitted. The probability of success for subsystem $i$ is denoted by $\pi_{i}=1-\theta_{i}$.

Second, order the subsystems (modules) according to the optimal testing procedure for series systems, so in non-decreasing order of $\frac{\kappa_{i}}{\theta_{i}}$. Denote the obtained order by $\sigma$; the optimal order is then given by $\left(L_{[1]_{\sigma}}, L_{[2]_{\sigma}}, \ldots, L_{[m]_{\sigma}}\right)$. Testing of a subsystem stops as soon as one working component has been found; testing of the system halts as soon as one subsystem has failed or all subsystems are known to be successful. Only in the latter case, does the overall system function.

\subsection{Reliability importance}

An interesting concept from the testing literature is the reliability importance of a component, first defined by Birnbaum [3] and used in exact testing algorithms, such as the one by Ben-Dov [1], and in approximation testing algorithms, such as by Jędrzejowicz [9]. In Section 4.2, we will use this concept in the development of a heuristic procedure for MP1.

Let the reliability function $h$ for a series-parallel system with system function as in (2) be the probability that the system will be working contingent on $\mathbf{p}$, so

$$
h(\mathbf{p})=\mathbb{E}[f(\mathbf{X})]=\prod_{i \in M}\left(1-\prod_{k \in N_{i}} q_{k}\right)=\prod_{i \in M} \pi_{i} .
$$

Following Birnbaum [3], we define the reliability importance $I_{k}$ of component $k$ as the partial derivative of $h$ with respect to $p_{k}$; this is a measure for the component's importance in determining whether the system will be up or down. If $I_{k}$ is zero then the component is irrelevant. The larger $I_{k}$, the more crucial or relevant the component to overall system success. Assuming $k \in N_{j}$, we obtain:

$$
I_{k}(\mathbf{p}) \equiv \frac{\partial h}{\partial p_{k}}(\mathbf{p})=-\frac{\partial h}{\partial q_{k}}(\mathbf{p})=\prod_{\substack{i \in M \\ i \neq j}}\left(1-\prod_{l \in N_{i}} q_{l}\right) \prod_{\substack{l \in N_{j} \\ l \neq k}} q_{l}=\prod_{\substack{i \in M \\ i \neq j}} \pi_{i} \prod_{\substack{l \in N_{j} \\ l \neq k}} q_{l} .
$$

We conclude that a job will be more important when, relatively to the other modules, its module has a low probability of success, and when relatively to the other jobs in its module, the job itself has a high probability of success. This is intuitive: since all modules have to succeed, modules with low success probability are more important. Conversely, since within a module the success of one job is sufficient, jobs with high success probability are more important.

\section{A greedy heuristic}

In this section, we propose four different greedy heuristics for MP1, in increasing order of quality of the solutions produced. For ease of presentation and understanding, we present the algorithms sequentially from simplest to most complicated: each algorithm is an improvement of the previous one. The optimal two-stage procedure for USPST is not directly applicable to MP1 because of two major differences. First, the MP1 setting includes precedence constraints, so the ordering 


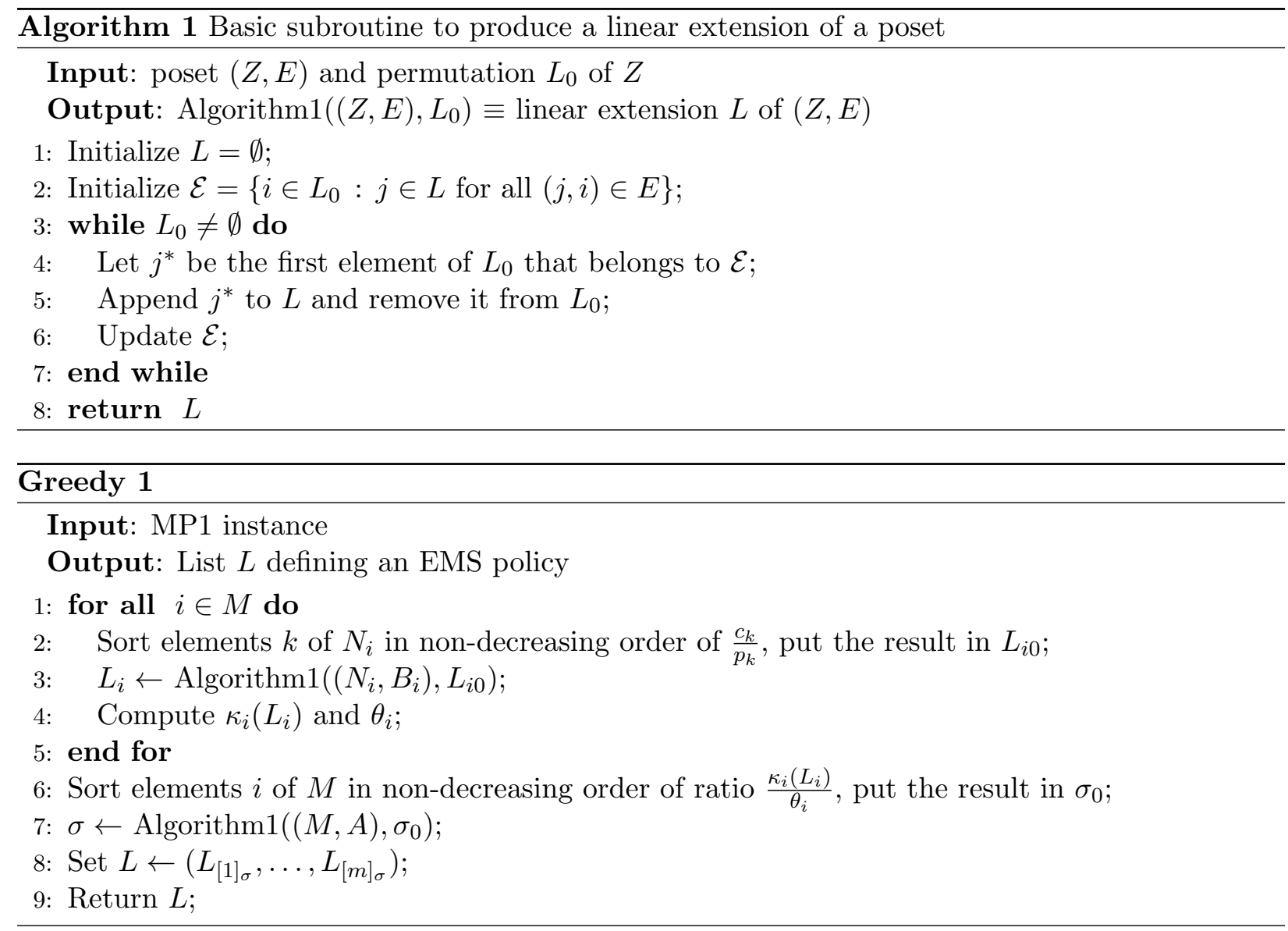

produced by the USPST algorithm may not be feasible. Second, the list defining an elementary testing policy contains all jobs, while job selection may be beneficial to the MP1 objective. The described procedures for MP1 are inspired by the exact algorithm for USPST, and we will consider EMS policies only, which can be conveniently represented by their compatible order list. Algorithm Greedy 1 generates an initial list of all jobs. Greedy 2 seeks to improve this initial solution by removing jobs from the list. Greedy 3 handles precedence constraints somewhat more intelligently than Greedy 1. In Greedy 4 we incorporate a randomization step to find better module orderings.

\subsection{Greedy 1: An initial list}

Algorithm 1 is a greedy procedure to transform a given permutation into a linear extension of a poset, thus producing a new permutation that respects the given set of precedence constraints. This procedure is used by the algorithm Greedy 1 to generate an initial order of all jobs.

To illustrate the functioning of this procedure, we consider the module depicted in Figure 3. Assume that in the absence of the precedence constraints, an optimal order within this module is $L_{i 0}=(3,2,4,5,1)$ (ordered in non-decreasing $\frac{c_{i}}{p_{i}}$ ). Greedy 1 then calls Algorithm 1 to transform $L_{i 0}$ into $L_{i}=(2,4,5,1,3)$.

\subsection{Greedy 2: Deciding which jobs not to schedule}

In order to decide which jobs not to include, we will compare the expected incremental benefit of scheduling a job with the expected incremental cost. The expected payoff when scheduling all jobs is $E P=h(\mathbf{p}) V$. Not scheduling job $k$ amounts to setting its $p_{k}$ equal to 0 . The probability vector 


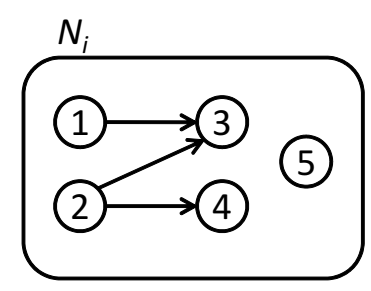

Figure 3: A module with precedence constraints between jobs.

then becomes $\mathbf{0}_{k} \circ \mathbf{p}$, where $\mathbf{0}_{k}$ denotes a vector with all 1 s except for a zero in position $k$, and ' $\circ$ ' the component-wise product. We derive the following first-order Taylor approximation:

$$
h(\mathbf{p}) \approx h\left(\mathbf{0}_{k} \circ \mathbf{p}\right)+\left(p_{k}-0\right) \frac{\partial h}{\partial p_{k}}\left(\mathbf{0}_{k} \circ \mathbf{p}\right) .
$$

By Equation (5), $\frac{\partial h}{\partial p_{k}}\left(\mathbf{0}_{k} \circ \mathbf{p}\right)=I_{k}\left(\mathbf{0}_{k} \circ \mathbf{p}\right)=I_{k}(\mathbf{p})$, and so the loss in expected payoff from removing job $k \in N_{j}$ is given by:

$$
\Delta E P=h(\mathbf{p}) V-h\left(\mathbf{0}_{k} \circ \mathbf{p}\right) V=p_{k} I_{k}(\mathbf{p}) V=\left(\prod_{\substack{i \in M \\ i \neq j}} \pi_{i} \prod_{\substack{l \in N_{j} \\ l \neq k}} q_{l}\right) p_{k} V .
$$

This result can be stated as an equality, since $h$ is linear in each of its arguments, so the first-order approximation of Equation (6) actually holds with equality.

We denote the expected cost by $E C$. Contrary to $E P$, the value of $E C$ depends on the order list at hand. Again, we will derive the incremental change compared to an initial list $L=\left(L_{[1]_{\sigma}}, \ldots, L_{[m]_{\sigma}}\right)$ of all jobs, generated for instance by Greedy 1 . For a module $j$, the following expected cost reduction will be obtained by removing the last job from $L_{j}$, say job $k$ :

$$
\Delta E C=\left(\prod_{i \in F(j)} \pi_{i} \prod_{\substack{l \in N_{j} \\ l \neq k}} q_{l}\right)\left(c_{k}+p_{k} \sum_{i \in G(j)}\left(\prod_{h \in G(j) \cap F(i)} \pi_{h}\right) \kappa_{i}\right),
$$

where $F(j)$ is the set of modules scheduled before module $j$ and $G(j)$ is the set of modules scheduled after $j$. Note that $\{F(j),\{j\}, G(j)\}$ is a partition of $M$, for any $j \in M$.

In conclusion, the net incremental expected profit of the removal of job $k$ that was scheduled last in a module $j$, is given by:

$$
\Delta E C-\Delta E P=\left(\prod_{i \in F(j)} \pi_{i} \prod_{\substack{l \in N_{j} \\ l \neq k}} q_{l}\right)\left(c_{k}+p_{k} \Gamma_{j}\right)-\left(\prod_{\substack{i \in M \\ i \neq j}} \pi_{i} \prod_{\substack{l \in N_{j} \\ l \neq k}} q_{l}\right) p_{k} V,
$$

where

$$
\Gamma_{j}=\sum_{i \in G(j)}\left(\prod_{h \in G(j) \cap F(i)} \pi_{h}\right) \kappa_{i}
$$

denotes the expected cost of the project after module $j$ is completed. If all modules before $j$ are successful, and all jobs in $N_{j}$ have failed, then if we are willing to forfeit the extra attempt offered 
by job $k$, not only will we save its own $\operatorname{cost} c_{k}$, but if job $k$ would have been successful, the expected cost of all modules further on in the schedule will no longer have to be laid out.

It seems preferable not to schedule a job when the net incremental expected profit given by Equation (7) is positive. To derive a conservative heuristic rule we underestimate $\Delta E C-\Delta E P$. This rule will be conservative in the sense that we are more likely to keep a job scheduled than in the apparent optimum. The reason is twofold: the heuristic rule obtained will be easier to interpret, and given the precedence constraints that have to be accounted for it is probably better to be conservative. Indeed, when a job is removed from the order list, all its successors need to be removed as well. Since this effect is very hard to factor in explicitly (and we did not in fact incorporate it in the derivations above), as a heuristic rule it will be better to be conservative about removing a job from the list.

Our negatively biased simplification of Equation (7) will consist in working with a smaller likelihood of saving the extra costs of the project from module $j$ onwards. To simplify notation, let

$$
\prod_{\substack{i \in M \\ i \neq j}} \pi_{i}=\alpha_{j}, \prod_{i \in F(j)} \pi_{i}=\beta_{j} \text { and } \prod_{i \in G(j)} \pi_{i}=\gamma_{j},
$$

with an empty index set resulting in a product of 1 . It holds that $\alpha_{j} \leq \beta_{j}, \alpha_{j}=\beta_{j} \gamma_{j}$ and

$$
\begin{aligned}
\Delta E C-\Delta E P=\beta_{j}\left(\prod_{\substack{l \in N_{j} \\
l \neq k}} q_{l}\right)\left(c_{k}+p_{k} \Gamma_{j}\right)-\alpha_{j}\left(\prod_{\substack{l \in N_{j} \\
l \neq k}} q_{l}\right) p_{k} V \\
\\
\geq \beta_{j}\left(\prod_{\substack{l \in N_{j} \\
l \neq k}} q_{l}\right) c_{k}-\alpha_{j}\left(\prod_{\substack{l \in N_{j} \\
l \neq k}} q_{l}\right) p_{k}\left(V-\Gamma_{j}\right) .
\end{aligned}
$$

We anticipate that it is better not to have job $k$ scheduled at the end of module $j$ if the right-hand side of the latter inequality (which is an underestimation of Equation (7)) exceeds zero, so if

$$
\beta_{j} c_{k} \geq \alpha_{j} p_{k}\left(V-\Gamma_{j}\right)
$$

With $\alpha_{j}=\beta_{j} \gamma_{j}$, we obtain the following heuristic selection rule:

$$
\frac{c_{k}}{p_{k}} \geq \gamma_{j}\left(V-\Gamma_{j}\right)
$$

That is, remove job $k \in N_{j}$ from the list if $\frac{c_{k}}{p_{k}}$ exceeds a fraction $\gamma_{j}$ of the difference between the project payoff $V$ and the expected further project cost $\Gamma_{j}$. Note that for instances with $|M|=1$ and $B_{1}=\emptyset$, this is exactly the optimal selection rule stated in (4).

In Greedy 2 we start from the list $L$ returned by Greedy 1, which contains all the jobs. Then, for each module, we remove all jobs that satisfy (9) from $L$ together with their successors, resulting in a shorter list $L^{\prime}$. Successor jobs must be removed as well to satisfy the precedence constraints. Removing jobs from a module $i$ increases $\theta_{i}$ and decreases $\kappa_{i}$, and thus module $i$ will have a lower ratio $\kappa_{i} / \theta_{i}$. Therefore, we may reorder the modules in $L^{\prime}$ by applying Algorithm 1 to the updated module order of non-decreasing ratio $\kappa_{i} / \theta_{i}$, resulting in another list $L^{\prime \prime}$; this latter list, however, does not necessarily lead to a better policy than the list $L^{\prime}$. There is also no guarantee that the selection process will improve the initial list $L$. Greedy 2 returns from the three lists $L, L^{\prime}$ and $L^{\prime \prime}$, one with the highest expected profit. 


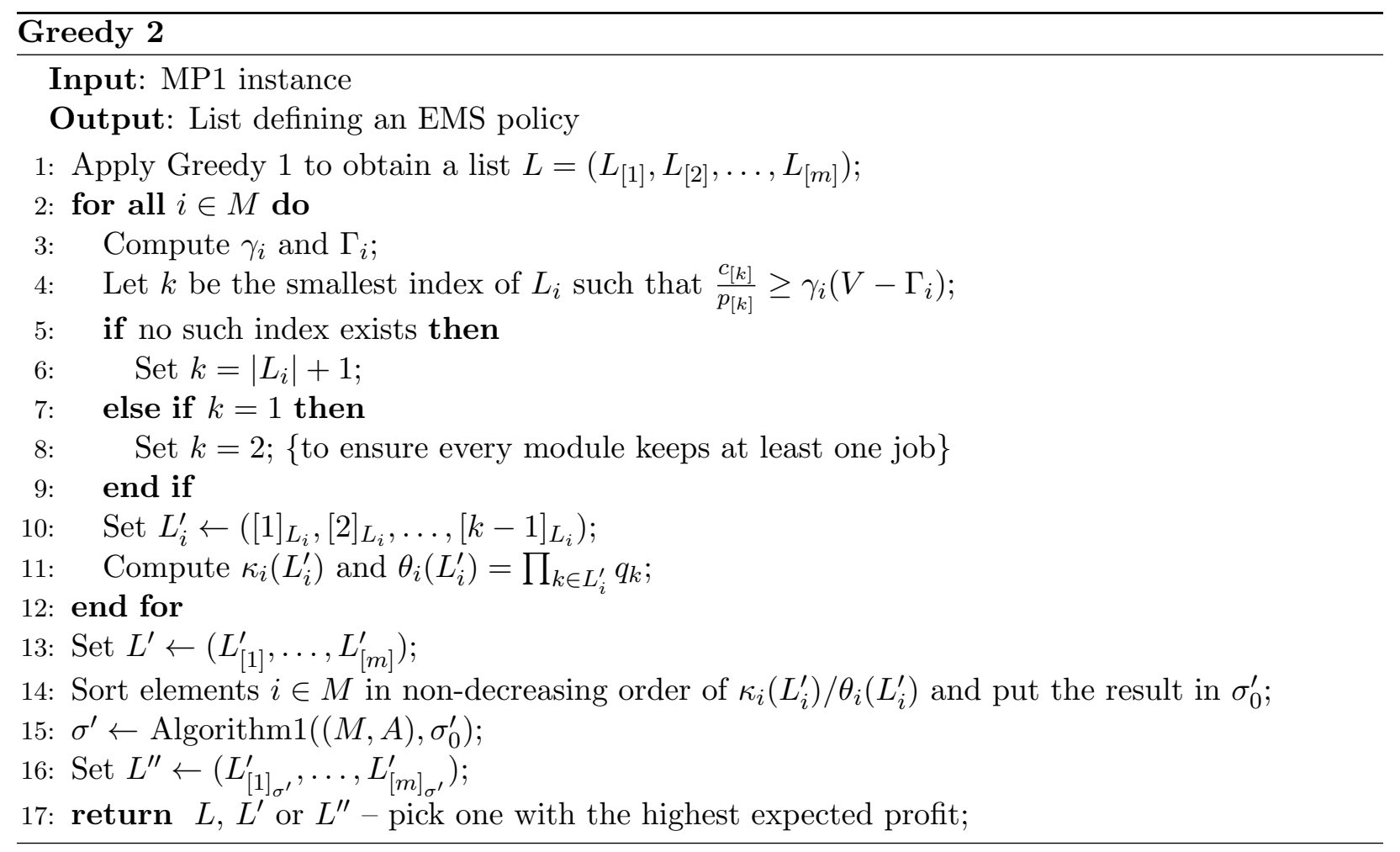

Table 1: A module with jobs that are not retained by Greedy 2.

\begin{tabular}{cccr}
\hline$k$ & $c_{k}$ & $p_{k}$ & $c_{k} / p_{k}$ \\
\hline 1 & 46 & 0.961 & 47.9 \\
2 & 10 & 0.891 & 11.2 \\
3 & 2 & 0.895 & 2.2 \\
4 & 12 & 0.836 & 14.4 \\
5 & 41 & 0.912 & 45.0 \\
\hline
\end{tabular}

Consider again the module depicted in Figure 3, for which Greedy 1 found the list $L_{i}=$ $(2,4,5,1,3)$. This module is part of a larger test instance, and part of the actual data are reproduced in Table 1 . Assume that $V=122, \gamma_{i} \approx 0.983$ and $\Gamma_{i} \approx 93.0$ (these values can only be determined on the basis of the entire instance, which is reproduced in Appendix A), then we have a critical value $\gamma_{i}\left(V-\Gamma_{i}\right) \approx 28.4$. Two ratios exceed this threshold: $\frac{c_{1}}{p_{1}}$ and $\frac{c_{5}}{p_{5}}$; job $5=[3]_{L_{i}}$ precedes job 1 in $L$. Note that, based on the functioning of Algorithm 1, the jobs after 5 in $L_{i}$ are either successors of 5 (such as 3 ) or have a more unfavorable ratio (such as 1 ). Consequently, starting from $L_{i}=(2,4,5,1,3)$, jobs 5,1 and 3 are removed, leading to the order $L_{i}^{\prime}=(2,4)$.

\subsection{Greedy 3: A refinement}

On studying the computational results of Greedy 1 and Greedy 2 (a detailed summary of these results is provided in Section 5), we observe that modules are frequently not in an optimal order because of the greediness of Algorithm 1. Consider the following example with three modules, $A=\{(1,2)\}$ and, for a certain order of their jobs, $\kappa_{1}=4, \kappa_{2}=1, \kappa_{3}=3$ and $\theta_{1}=0.2, \theta_{2}=$ $0.5, \theta_{3}=0.2$. The ratios $\kappa / \theta$ are 20,2 and 15 , and the desired order $\sigma_{0}=(2,3,1)$. Calling 


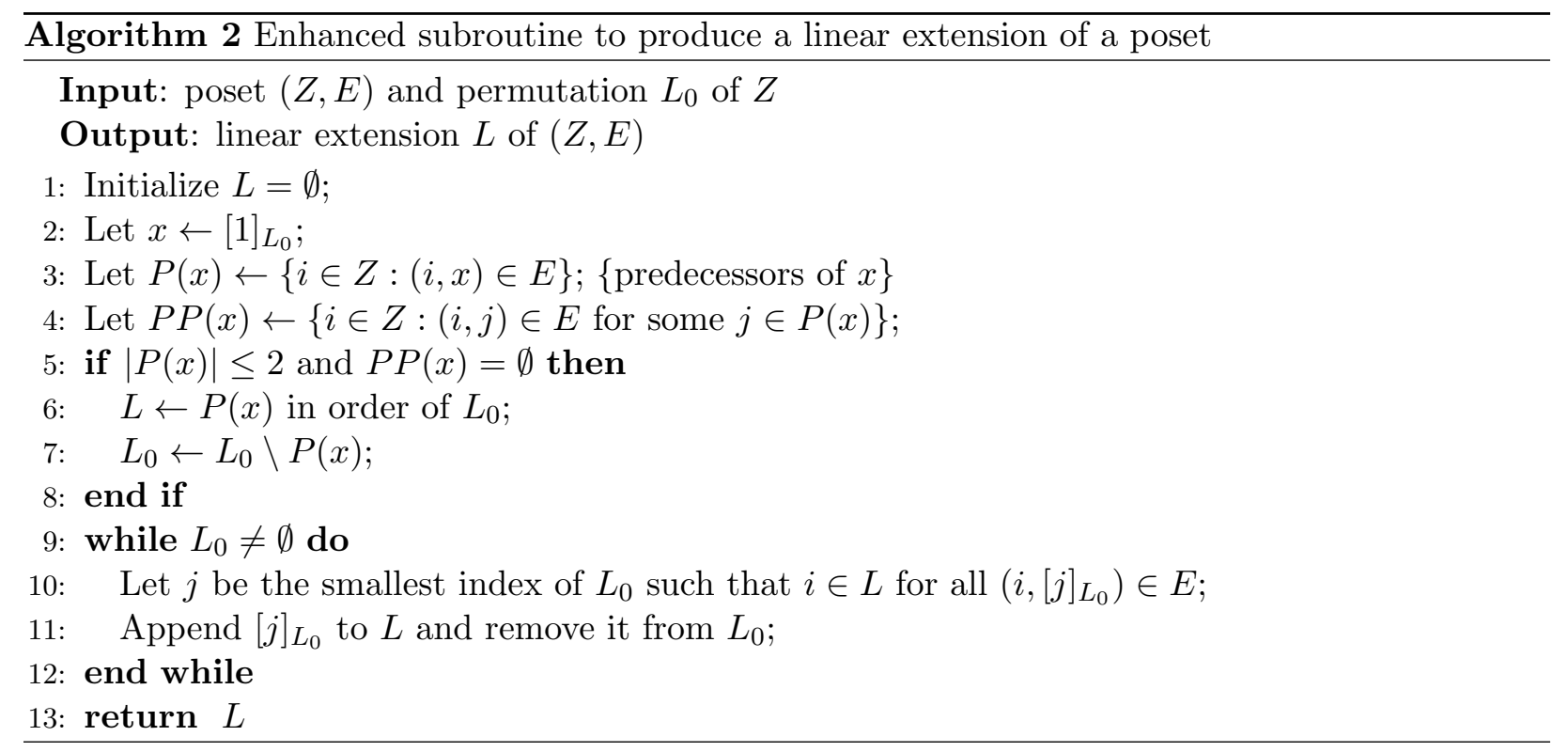

Algorithm1 $\left((M, A), \sigma_{0}\right)$ yields $\sigma=(3,1,2)$, with expected cost $\kappa_{3}+\pi_{3}\left(\kappa_{1}+\pi_{1} \kappa_{2}\right)=6.84$. Even though module 2 is clearly preferable and has a low cost, it cannot be placed first because module 1 is a predecessor. Algorithm 1 places module 3 first because module 1 has a ratio that is slightly higher than that of module 3 . It is an optimal decision, however, to select module 1 first in order to enable module 2 to come earlier. The order $(1,2,3)$ has (lower) expected cost $\kappa_{1}+\pi_{1}\left(\kappa_{2}+\pi_{2} \kappa_{3}\right)=6$.

The described effect will only be significant if the module with the lowest ratio, say module $j$, (a) has a ratio that is considerably lower than all the other modules, (b) has very few predecessors, (c) these predecessors have a ratio that is not too high relative to the modules other than $j$, (d) these predecessors do not have any predecessors themselves. In the example, (a) clearly holds since $2<<15$ and $2<<20$, (b) holds since module 2 has only one predecessor, (c) holds since 20 does not exceed 15 by orders of magnitude, and (d) holds since module 2 has no predecessors. To develop an improved version of Algorithm 1, we will check the number of predecessors of module $j$. If this is two or less (condition (b)) and they have no predecessors themselves (condition (d)) then we will try to order the predecessors to module $j$ first. The enhanced subroutine is described in Algorithm 2. Conditions (a) and (c) are hard to pin down, and so we will simply try both Algorithm 1 and Algorithm 2, and see which one performs best.

The enhanced subroutine can now be put to work in Greedy 3. It will only be used to order modules, not jobs. From the tests on the dataset, we observe that the optimal order of jobs within a module is nearly always obtained, and if not then the loss in quality is usually not very important. We conclude by noting that a problem would arise if we were to apply this subroutine also within modules: a choice would need to be made to which modules to apply Algorithm 2 and to which Algorithm 1, either heuristically, or by checking all possibilities - which would make the runtime of the heuristic exponential in the number of modules.

\subsection{Greedy 4: Randomizing the module order}

The quality of the heuristic selection rule derived for Greedy 2 highly depends on the ordering of the modules and only works well when the module order is (near-)optimal. In the previous subsection we have illustrated that the simple greediness of Algorithm 1 may result in module orderings that are far from optimal. For this reason, Greedy 3 orders the modules in a slightly more enhanced manner; improvements, however, are realized only in very specific situations. To 


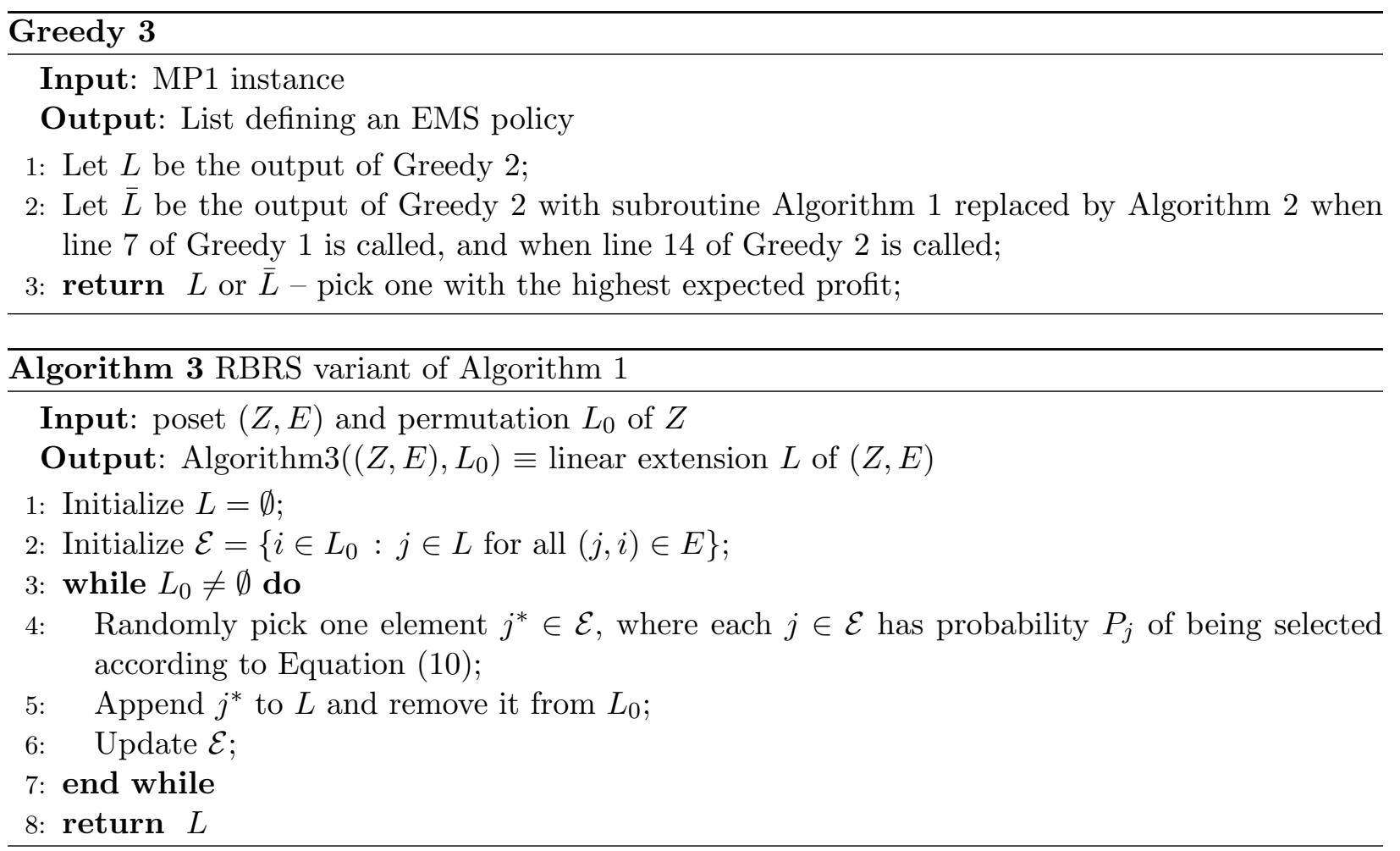

overcome this, we have introduced a randomization step in order to increase the likelihood of finding good module orderings. Instead of always choosing from the set $\mathcal{E}$ of eligible modules one with lowest ratio $\kappa / \theta$ (see Algorithm 1 ), we now create the possibility of selecting a module with a higher ratio (Algorithm 3) by means of regret-based random sampling (RBRS) [8]. The probability $P_{i}$ of selection of a module $i$ out of the set $\mathcal{E}$ is determined by means of priority values $v$, as follows:

$$
P_{i}=\frac{\left(\rho_{i}+1\right)^{\alpha}}{\sum_{j \in \mathcal{E}}\left(\rho_{j}+1\right)^{\alpha}},
$$

with $\rho_{i}=\left(\max _{j \in \mathcal{E}} v_{j}\right)-v_{i}$. In Greedy 4 , we start from the result of Greedy 3 to guarantee producing a solution that is at least as good. Subsequently, we invoke Greedy 2 for different module orderings obtained via Algorithm 3 until a stopping criterion is met.

In our implementations, the priority value $v_{i}$ of module $i$ is chosen to be the position of module $i$ in the initial module ordering $L_{0}$ (non-decreasing $\kappa / \theta$ ) rather than the ratio $\kappa / \theta$ itself, because the ratio can take rather extreme values (especially for large modules). The parameter $\alpha \in[0, \infty)$ controls the diversification of the sample of module orderings selected from the population of all possible module orderings. The diversification in the module lists decreases with $\alpha$. The boundary case of $\alpha=0$ corresponds to a completely random selection from $\mathcal{E}$ with equal probability $1 /|\mathcal{E}|$, whereas in the other extreme of $\alpha=+\infty$, we always select that element of $\mathcal{E}$ appearing first in the initial ordering $L_{0}$ (as in Algorithm 1). In Section 5.1 we elaborate on the choice of the parameter $\alpha$ and on the stop criterion.

\section{Computational experiments}

In this section, we assess the performance of the algorithms on the same dataset ${ }^{1}$ of 360 instances $^{2}$ that is also used by Coolen et al. [5]. The instances are indexed according to their size, mea-

\footnotetext{
${ }^{1}$ available online at www.econ.kuleuven.be/public/NDBAC96/MP1_instances.htm
} 


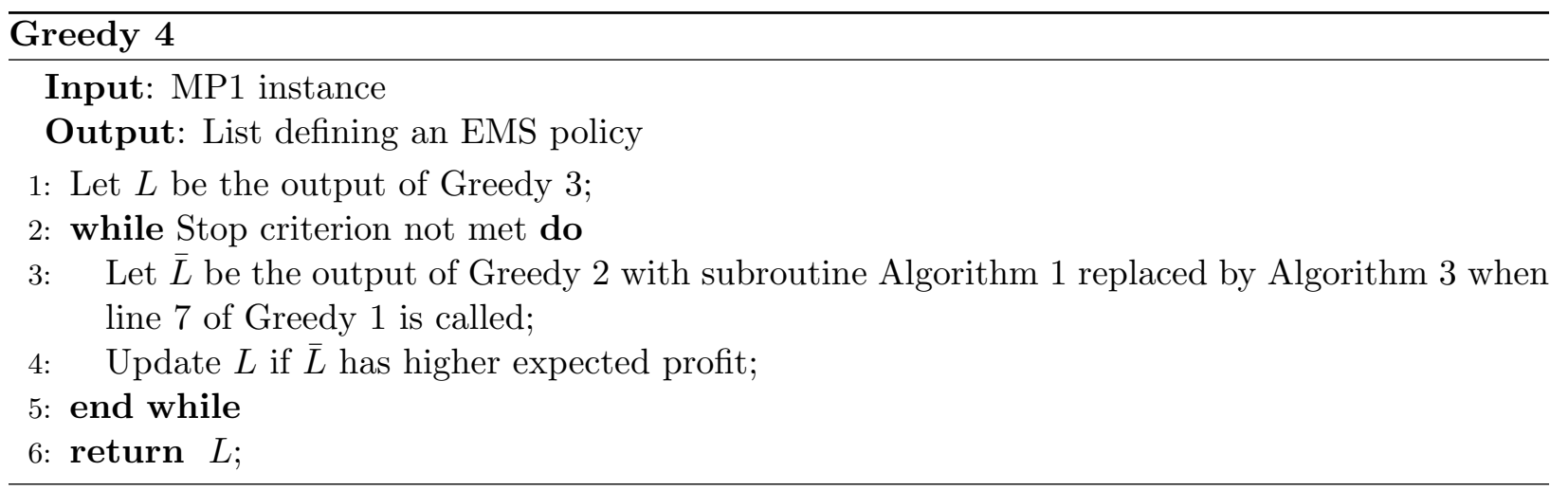

sured by the number $n$ of jobs, and their order strength $O S$. The order strength is defined as the number of precedence-related activity pairs in the induced network $\left(N, B^{*}\right)$ divided by the maximum possible number of such pairs, and therefore equals $\left|B^{*}\right| /\left(\begin{array}{l}n \\ 2\end{array}\right)$. For each combination of $n \in\{10,20,30, \ldots, 120\}$ and $O S \in\{0.4,0.6,0.8\}, 10$ instances were created. Full details on the data generation can be found in the predecessor paper.

All experiments were run on a Dell Latitude D830 with a $2.5 \mathrm{GHz}$ processor and $3 \mathrm{~GB}$ of RAM, running Windows Vista 32 bits. The algorithms were implemented in $\mathrm{C}++$ using Microsoft Visual Studio 2010. The performance of our heuristics was evaluated against the two exact algorithms developed in [5]: a branch-and-bound (B\&B) algorithm that finds an optimal EMS policy, and a dynamic-programming (DP) algorithm that outputs a globally optimal policy. To facilitate comparison, we define the relative optimality gap $R O G$ of an algorithm $\mathcal{A}$ for an instance of MP1 as follows:

$$
R O G=\frac{z^{*}-z(\mathcal{A})}{z^{*}} \text { if } z^{*} \neq 0, \text { and } 0 \text { otherwise, }
$$

with $z^{*}$ the objective value of a globally optimal solution (found by DP) and $z(\mathcal{A})$ the objective of the output of $\mathcal{A}$.

Below, we include a discussion of some implementation choices for heuristic Greedy 4 (Section 5.1), followed by a presentation of the computational results (Section 5.2).

\subsection{Implementation choices for Greedy 4}

For the implementation of algorithm Greedy 4, we need to decide on the stop criterion and on the value of the parameter $\alpha$. Reaching a maximum number $\mu_{\max }$ of different module orders allowed to be generated by Algorithm 3, is a natural stop criterion. In Figure 4 we show the relative optimality gap in function of $\mu_{\max }$ for different values of $\alpha$, for a small instance with 20 jobs (Figures $4(\mathrm{a})$ and 4(b)), and for a large instance with 120 jobs (Figures 4(c) and 4(d)). For each instance the left plot focuses only on small values of $\mu_{\max }$ (at most 50), whereas the right plot shows the performance of Greedy 4 when more module orderings are generated (at most 1000).

The performance of Greedy 4 on the dataset was assessed with two different stop criteria. Firstly, when it is desirable that the computation time for Greedy 4 be of the same order of magnitude as Greedy 1, Greedy 2, and Greedy 3 (i.e., only a fraction of a second), we set $\mu_{\max }=50$. We will refer to this variant as Greedy 4a. When finding a higher-quality solution is important but still aiming to develop a fast heuristic, a time limit of one second is imposed as the second stop criterion; this variant of the algorithm is named Greedy $4 \mathrm{~b}$. We can see from Figure 4 that a convenient choice for the parameter $\alpha$ depends on the stop criterion selected.

In Greedy 4a, only a low number of (different) module orderings are generated. Typically, the total number of possible module orderings is far higher than $\mu_{\max }=50$, and good orderings need to 


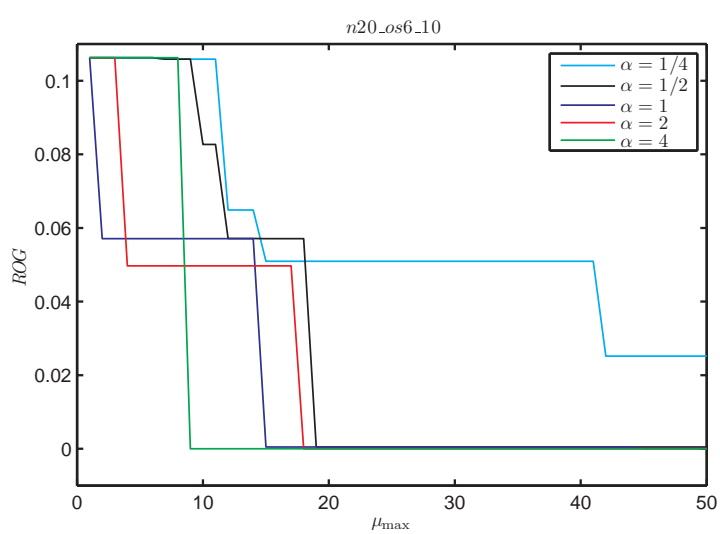

(a) $n=20,1 \leq \mu_{\max } \leq 50$

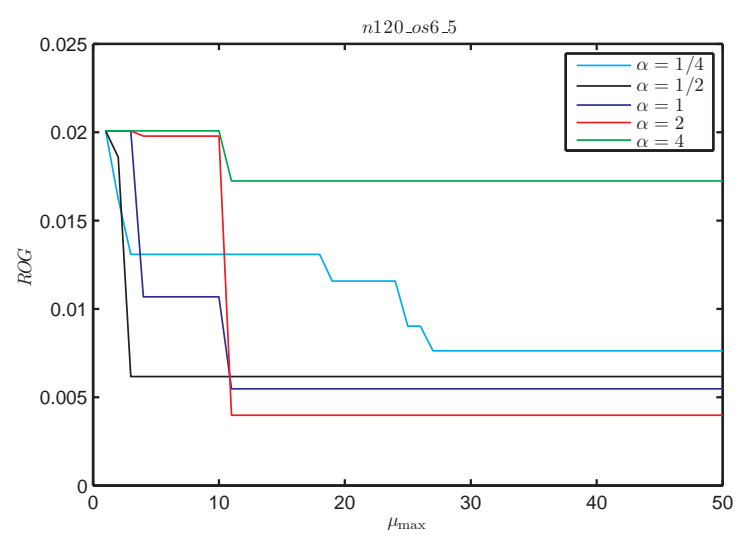

(c) $n=120,1 \leq \mu_{\max } \leq 50$

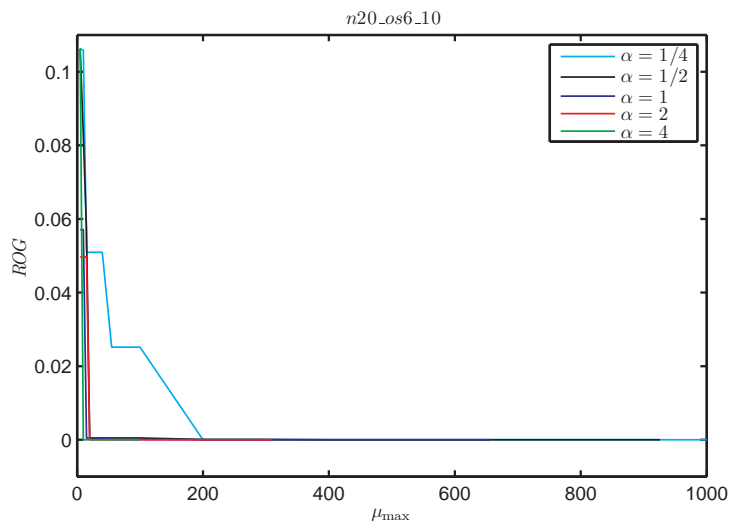

(b) $n=20,1 \leq \mu_{\max } \leq 1000$

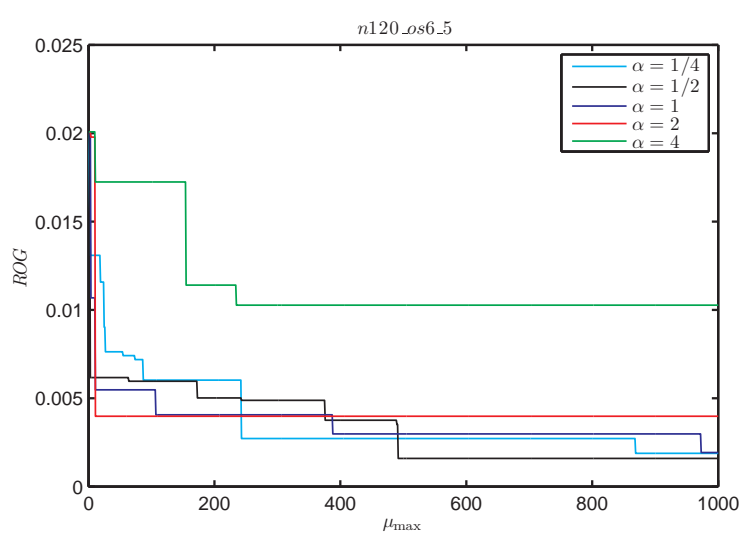

(d) $n=120,1 \leq \mu_{\max } \leq 1000$

Figure 4: Relative optimality gap in function of $\mu_{\max }$ for $\alpha \in\{1 / 4,1 / 2,1,2,4\}$ of a small instance with 20 jobs (n20_os6_10) and a large instance with 120 jobs (n120_os6_5). The left figures only show $\mu_{\max } \leq 50$, the right figures have $\mu_{\max } \leq 1000$.

be found with only a few attempts (see Figure 4(c)). It is to be expected that orderings that do not differ too much from the ordering generated by Algorithm 1 are likely to be of reasonable quality. Figure 4(c) shows that too much diversification $(\alpha=1 / 4,1 / 2)$ will lead to worse solutions compared to a lower diversification level $(\alpha=1,2)$. It is also crucial, however, to allow for a certain level of flexibility in the orderings, as is illustrated by the poor performance of $\alpha=4$. From Figure 4(a) we observe that the performance of Greedy 4 is less sensitive to the level of diversification when the instance is small. This is to be expected: the total number of module orderings is also far lower. In general we infer that $\alpha=2$ is a good choice for Greedy 4 a.

Greedy 4b works with a time limit of one second; within this time we can produce close to 2000 different module orders for the large instances. Since more lists can be generated, we expect a higher diversification level to perform better; Figure 4(d) suggests $\alpha=1 / 2$ as the best choice. It is to be noted that for the small instance of 20 jobs we find only 1000 different orders for $\alpha=1 / 4$ (within a time limit of one second). Higher values of $\alpha$ result in a high number of duplicate orderings: when $\alpha=4$, for example, we encounter only 97 different module lists (within one second).

\subsection{Computational results}

In Table 2 the average relative optimality gaps are given for the instances with $n \in\{10,20,30,40\}$. The B\&B cannot solve all the corresponding 120 instances to guaranteed optimality within a time 
Table 2: Average performance over 85 instances with $n \in\{10,20,30,40\}$.

\begin{tabular}{llrr}
\hline Algorithm & Resulting policy & CPU (s) & $R O G$ \\
\hline DP & Global optimum & $<0.01$ & $0.00 \%$ \\
B\&B & Optimal EMS & 81.28 & $0.01 \%$ \\
Greedy 4b & Randomized heuristic EMS & 1.00 & $0.13 \%$ \\
Greedy 4a & Randomized heuristic EMS & 0.66 & $0.18 \%$ \\
Greedy 3 & Deterministic heuristic EMS & $<0.01$ & $1.97 \%$ \\
Greedy 2 & Deterministic heuristic EMS & $<0.01$ & $3.21 \%$ \\
Greedy 1 & Deterministic heuristic EMS & $<0.01$ & $4.76 \%$ \\
\hline
\end{tabular}

limit of 30 minutes; especially larger instances and instances with a lower $O S$ are more difficult to solve. We therefore report the average performance only for the 85 instances solved by the B\&B. The average CPU time is less than 0.01 seconds for the DP algorithm and for the three deterministic greedy algorithms. The average CPU time for Greedy $4 \mathrm{a}$ is 0.66 seconds: some time is needed for generating $\mu_{\max }$ different module orders (or for reaching a time limit of one second, whichever comes first) - this will not be the case for larger instances (see infra). For the 85 instances solved by the $\mathrm{B} \& \mathrm{~B}$, the average $R O G$ is about $0.01 \%$, so the expected profit is virtually the same in each case. The $R O G$ of our final heuristic Greedy $4 \mathrm{~b}$ is $0.13 \%$. When only 50 module orderings are generated, the gap increases by $0.05 \%$ up to $0.18 \%$. For our best deterministic heuristic (Greedy 3), the gap is significantly higher than for Greedy $4(1.97 \%)$. Greedy 2, with a simpler ordering subroutine, achieves $3.21 \%$ and the average gap of Greedy 1 is yet slightly higher at $4.76 \%$. This means that the average extra profit achieved by making a selection of jobs amounts to $1.55 \%$ of the global optimum. Looking into the dataset in more depth, we observe that in 13 instances the optimal EMS policy generated by B\&B does not schedule all available jobs. In seven out of these, Greedy 3 makes the same selection. In three instances, Greedy 3 makes a selection that is somewhat larger than the optimal one. For three other instances, Greedy 3 fails to make a selection and schedules all available jobs, while B\&B finds an optimal policy that only schedules a subset of $N$. Greedy $4 \mathrm{~b}$ finds the optimal EMS policy for all but one of these 13 instances.

For the instances with $n>40$, the results of the heuristic can only be compared to the DP, which can solve 171 out of the 240 remaining instances without memory overrun; the results for these 171 instances are summarized in Table 3. The unsolved instances are again those with high $n$ and low $O S$. The average runtime of the DP increases significantly when the instances become larger: it now needs 83.07 seconds on average; especially instances with a low order strength need more time. The runtime of each greedy algorithm is less than 0.01 seconds, except for Greedy 4b (which has an imposed time limit). Compared to Table 2, we observe that Greedy $4 \mathrm{a}$ is significantly faster for these larger instances: finding 50 different orderings turns out to be far easier for higher $n$. The gaps for Greedy 1, 2 and 3 are slightly lower than for the instances solved by B\&B (Table 2). A possible explanation might be that the optimal objective value increases with $n$ (which is inherent in the way the instances were created). The improvement of Greedy 2 vis-à-vis Greedy 1 is substantially smaller, at a mere $0.18 \%$. Since optimal EMS policies are not available for these instances, we cannot assess whether this is because our heuristic selection rule (Equation (9)) performs worse for larger instances, or because the instances in this part of the dataset simply did not require a selection of jobs. The improvement realized by Greedy 4 is smaller for the small instances (Table 2) than for the larger instances (Table 3), but the average relative optimality gap for our best heuristic, Greedy $4 \mathrm{~b}$, is still small (only a half percent).

For the instances that were not solved by the DP, we cannot determine $R O G$ because a global 
Table 3: Average performance over 171 instances with $n \in\{50,60, \ldots, 120\}$.

\begin{tabular}{llrr}
\hline Algorithm & Resulting policy & CPU (s) & $R O G$ \\
\hline DP & Global optimum & 83.07 & $0.00 \%$ \\
Greedy 4b & Randomized heuristic EMS & 1.00 & $0.50 \%$ \\
Greedy 4a & Randomized heuristic EMS & $<0.01$ & $0.94 \%$ \\
Greedy 3 & Deterministic heuristic EMS & $<0.01$ & $1.64 \%$ \\
Greedy 2 & Deterministic heuristic EMS & $<0.01$ & $1.67 \%$ \\
Greedy 1 & Deterministic heuristic EMS & $<0.01$ & $1.85 \%$ \\
\hline
\end{tabular}

optimum is not known. In this case, we can only evaluate the relative gap of our algorithms with respect to the best solution found, i.e. the expected profit found by Greedy 4b. Column C1 of Table 4 contains the average gaps for the heuristics compared to Greedy $4 \mathrm{~b}$ over the 69 instances that were not solved by DP. We observe an average improvement with respect to Greedy 1 and Greedy 2 of $0.52 \%$. The selection rule seems to fail in Greedy 2 (no improvement by selection in any of these 69 instances). The improvement of Greedy 3 and Greedy 4 is realized by a reordering of the modules. Here also, no selection of jobs occurs; again we cannot evaluate whether this is because the selection rule of Equation (9) becomes of lesser quality for higher values of $n$, or rather because no selection is required. Column $\mathrm{C} 2$ in Table 4 reports the averages over all 360 instances of the dataset.

Figure 5 depicts the $R O G$ of Greedy $4 \mathrm{~b}$ as a function of $n$, for each of the three values of $O S$. The curves apply to the 289 instances solved by DP; each observation is the average for the solved instances of the setting considered (at most 10). No clear patterns arise; the significant fluctuations are presumably due simply to random idiosyncrasies in the dataset.

\section{Conclusions}

This paper has looked into the problem of modular project scheduling on one machine (MP1); exact scheduling algorithms for this type of projects have recently been developed by Coolen et al. [5] . When only few precedence constraints are imposed, however, these algorithms either run out of memory or become very slow when the number of activities increases. The goal of this paper was to develop a heuristic that produces 'good' schedules for such projects, i.e., schedules with a high expected profit, while requiring only very limited CPU time and computer memory.

MP1 is closely related to the series-parallel sequential testing problem. The optimal two-step procedure for the testing problem without precedence constraints is the starting point for the

Table 4: Average relative improvement of Greedy 4b over the remaining 69 instances that were not solved by DP (column C1), and over all 360 instances of the dataset (column C2).

\begin{tabular}{lrr}
\hline Algorithm & C1 & C2 \\
\hline Greedy 4b & $0.00 \%$ & $0.00 \%$ \\
Greedy 4a & $0.26 \%$ & $0.38 \%$ \\
Greedy 3 & $0.46 \%$ & $1.32 \%$ \\
Greedy 2 & $0.52 \%$ & $1.72 \%$ \\
Greedy 1 & $0.52 \%$ & $2.18 \%$ \\
\hline
\end{tabular}




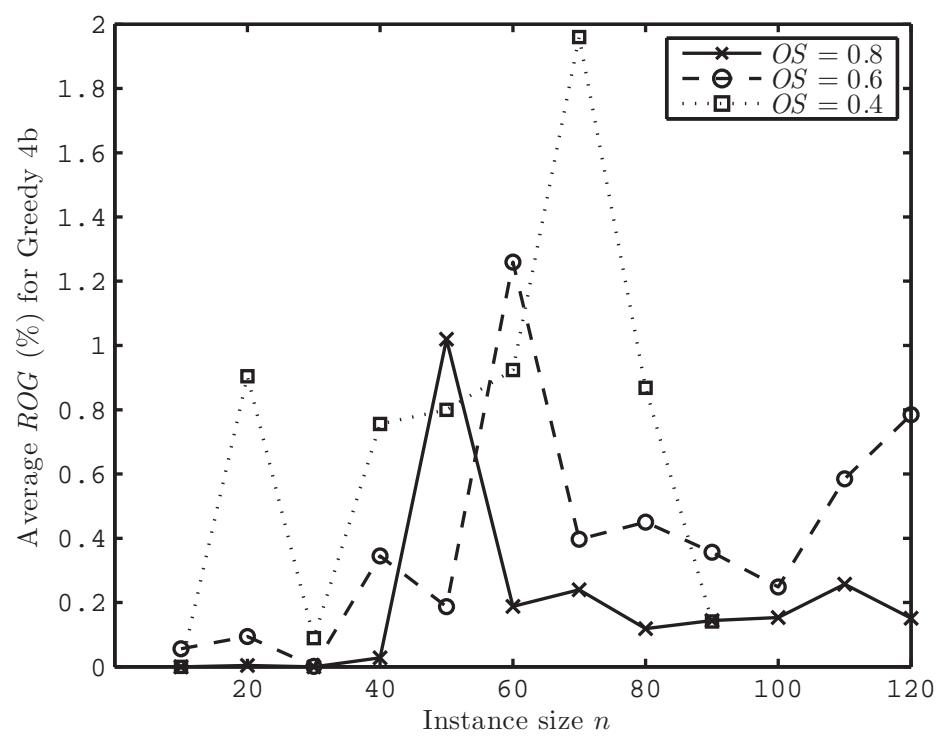

Figure 5: Average relative optimality gap of Greedy 4b.

development of a fast heuristic procedure for MP1, in which we essentially produce an order list that defines a so-called 'elementary module-sequence' scheduling policy. Four variants are proposed, in increasing order of complexity. The starting point is a simple greedy algorithm, and this is stepwise refined and complemented with a randomization step in the final variant. Based on computational experiments on a large dataset, we find that the algorithms output good solutions in negligible runtimes: the average optimality gap for instances for which an optimal solution is known, is quite limited $(0.5 \%$ or less). We produce approximate solutions for complex instances that could not be tackled by an exact algorithm in the earlier reference, and this within runtimes of at most one second.

A possible avenue for further research on the topic of modular project scheduling is the exploration of the multi-mode character of the modules: every module can also be seen as a 'composite' activity, which can be executed in multiple 'modes' - each mode corresponding to one possible selection of activities within the module. The literature on (especially project) scheduling has already looked into various multi-mode problems (e.g., time-cost, time-resource and resource-resource trade-offs) but, to the best of our knowledge, multiples modes corresponding to multiple selections of (sub-)activities contained in the original activity (in our case: the module) have not yet been described in earlier work. From an algorithmic viewpoint, an obvious further step towards finding high-quality solutions to MP1 would be the development of a meta-heuristic procedure (for instance, tabu search or genetic algorithms, or any other meta-heuristic framework); in light of the low optimality gaps that are already achieved by the (faster) algorithms proposed in this paper, however, we have not pursued this option.

\section{Appendix A An instance with $n=20$}

In this appendix, for illustration purposes, we describe the outputs of the algorithms proposed in this text for the instance depicted in Figure 6. The numerical data for this instance can be found in Table 5. The instance is part of the dataset (instance name g_n20_os6_4).

The initial ordering generated by Greedy 1 is

$$
L=(2,4,5,1,3 ; 6,7 ; 19,20 ; 8,9,10,11,12 ; 13,15,16,17,14,18),
$$




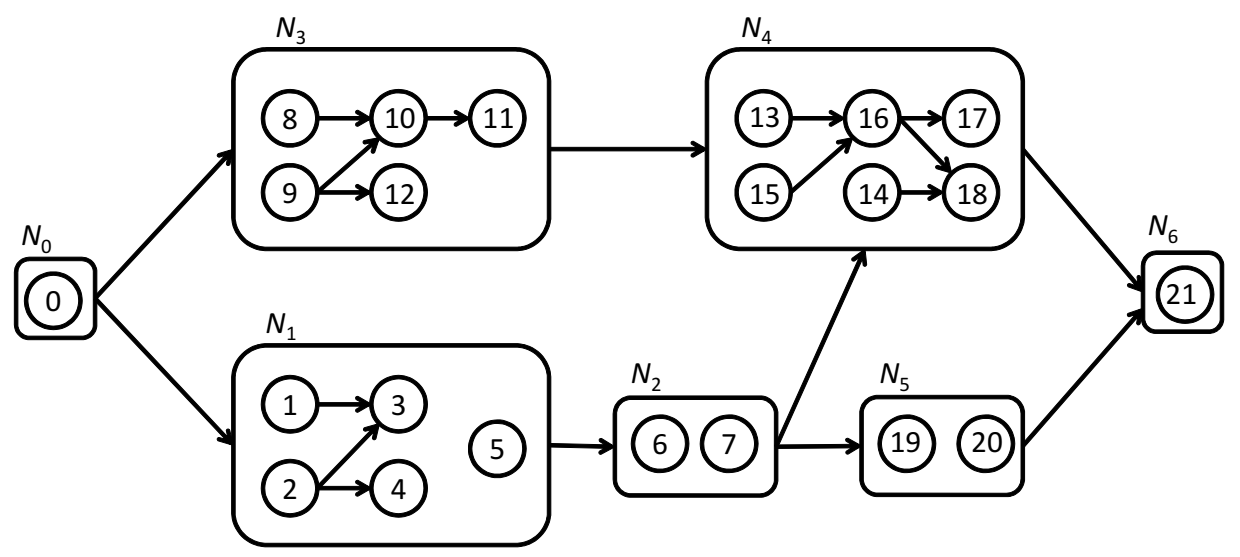

Figure 6: Precedence network.

Table 5: Costs and probabilities.

\begin{tabular}{lrrrrrrrrrr}
\hline$k$ & 1 & 2 & 3 & 4 & 5 & 6 & 7 & 8 & 9 & 10 \\
$c_{k}$ & 46 & 10 & 2 & 12 & 41 & 32 & 33 & 15 & 41 & 16 \\
$p_{k}$ & 0.961 & 0.891 & 0.895 & 0.836 & 0.912 & 0.977 & 0.844 & 0.833 & 0.922 & 0.978 \\
$c_{k} / p_{k}$ & 47.9 & 11.2 & 2.2 & 14.4 & 45.0 & 32.8 & 39.1 & 18.0 & 44.5 & 16.4 \\
\hline$k$ & 11 & 12 & 13 & 14 & 15 & 16 & 17 & 18 & 19 & 20 \\
$c_{k}$ & 15 & 24 & 17 & 46 & 22 & 33 & 45 & 42 & 14 & 41 \\
$p_{k}$ & 0.972 & 0.903 & 0.856 & 0.825 & 0.860 & 0.966 & 0.902 & 0.906 & 0.898 & 0.866 \\
$c_{k} / p_{k}$ & 15.4 & 26.6 & 19.9 & 55.8 & 25.6 & 34.2 & 49.9 & 46.4 & 15.6 & 47.3 \\
\hline$V$ & 122 & & & & & & & & & \\
\hline
\end{tabular}

which is easy to verify manually with the data from Table 5 and the pseudocode of Greedy 1 . Greedy 2 removes jobs 1,3 and 5 and the order of the modules is redetermined. In this case, the heuristic order of the modules does not change $\left(L^{\prime}=L^{\prime \prime}\right)$, and

$$
L^{\prime}=(2,4 ; 6,7 ; 19,20 ; 8,9,10,11,12 ; 13,15,16,17,14,18) .
$$

The expected profit from $L$ is approximately 14.72, from $L^{\prime} 15.05$, so Greedy 2 will return $L^{\prime}$. Greedy 3 finds the same solution. An optimal EMS policy found via B\&B turns out to be slightly better, with an expected profit of 15.32. An optimal ordering is given by

$$
(8 ; 2,4,5,1,3 ; 6,7 ; 19,20 ; 13,15,16,17,14,18) ;
$$

this list is also found by the two implementations of Greedy 4.

\section{References}

[1] Y. Ben-Dov. A branch and bound algorithm for minimizing the expected cost of testing coherent systems. European Journal of Operational Research, 7(3):284-289, 1981.

[2] Y. Ben-Dov. Optimal testing procedures for special structures of coherent systems. Management Science, 27(12):1410-1420, 1981. 
[3] Z.W. Birnbaum. On the importance of different components in a multi-component system. In P.R. Krishnaiah, editor, Multivariate Analysis-II, volume 1913, pages 15-26. Academic Press, New York, 1969.

[4] R.W. Butterworth. Some reliability fault-testing models. Operations Research, 20(2):335-343, 1972 .

[5] K. Coolen, W. Wei, F. Talla Nobibon, and R. Leus. Project scheduling with modular project completion on a bottleneck resource. Journal of Scheduling, to appear. DOI: 10.1007/s10951012-0294-9.

[6] B. De Reyck, Y. Grushka-Cockayne, and R. Leus. A new challenge in project scheduling: The incorporation of activity failures. Review of Business and Economics, LII(3):411-434, 2007.

[7] B. De Reyck and R. Leus. R\&D-project scheduling when activities may fail. IIE Transactions, 40(4):367-384, 2008.

[8] A. Drexl. Scheduling of project networks by job assignment. Management Science, 37(12):1590-1602, 1991.

[9] P. Jędrzejowicz. Minimizing the average cost of testing coherent systems: Complexity and approximate algorithms. IEEE Transactions on Reliability, R-32(1):67-70, 1983.

[10] L.G. Mitten. An analytic solution to the least cost testing sequence problem. The Journal of Industrial Engineering, page 17, January-February 1960.

[11] T. Ünlüyurt. Sequential testing of complex systems: A review. Discrete Applied Mathematics, 142:189-205, 2004. 